\title{
Animalische Lizenzen
}

\section{Zur Artikulation und Reflexion von Ordnung in Johann Fischarts Flöh Hatz / Weiber Tratz und Georg Rollenhagens Froschmeuseler}

\author{
Was hör ich aus dem Winkel dort \\ Für ain gschrai / was kläglich wort? \\ [...] \\ Aber Boz Laus / es ist der Floch / \\ Wie komts? er springt jz nicht so hoch / \\ [...] \\ Ich glaub im sei ain bain entzwai / \\ Er führt wol so ain jamergschrai / \\ Wiwol er sonst schweigt allezeit / \\ Weil schreien nicht dint zu seim streit / \\ So gfrirt im jz der Schnabel auf: \\ Gewiß bedeits kain guten kauf: \\ Dan wie die Wunderbücher setzen / \\ Bedeits nichts guts / wan die Thir schwetzen: ${ }^{1}$
}

Selten bedeutet es etwas Gutes, wenn Tiere zu schwetzen anfangen. Entweder, so heißt es zu Beginn von Johann Fischarts Flöh Hatz Weiber Tratz, hat man es mit aus ,Wunderbüchern“ übermittelten Prodigien zu tun, die gegen die natürliche Schöpfungsordnung verstoßen und daher Schlimmes verheißen. Oder Tiere erheben ihre Stimme, wenn, etwa im Angesicht des Metzgers, nun der Tod sich fügt (FH 68) und die Existenz bedroht ist.

Fischart markiert hier die alltagsweltliche, menschliche Sicht auf die Tierstimme; dass wir es zugleich mit einer weiteren Form animalischer Rede zu tun haben, wird indessen daran deutlich, dass sich hier kein menschlicher Sprecher äußert, sondern eine Fliege. Fischart greift somit auf das Register der Tierfabel zurück, in der es gerade nicht die wundersame und beunruhigende Ausnahme, sondern vielmehr die Regel darstellt, dass Tiere sprechen. Die Fabel beruht auf

1 Fischart, Johann: Flöh Hatz, Weiber Tratz. Hrsg. von Alois HAAS, Stuttgart 1967 (RUB 1656/ 56a), 48-64. Im Folgenden unter der Sigle FH.

Prof. Dr. Julia Weitbrecht, Universität zu Köln, Institut für Deutsche Sprache und Literatur I, Ältere deutsche Sprache und Literatur, Albertus Magnus Platz, 50923 Köln, j.weitbrecht@uni-koeln.de 
dieser „programmatischen Unwahrscheinlichkeit“2, denn sie entwirft eine Welt, in der Menschen und Tiere völlig selbstverständlich auf einer Ebene miteinander kommunizieren.

Nur wenige literarische Formen verfügen über eine in historischer Perspektive so große Stabilität wie die Fabel. Ihr Arsenal an nicht-menschlichen Akteuren sowie die damit verbundene Auslegungstradition, sie auf das Verhalten der Menschen zu beziehen, bilden seit der griechischen Antike die Konstanten der Fabelpoetik. Zugleich bietet die Fabel in ihrer flexiblen Verbindung von Erzählung und Auslegung ein reiches Spektrum an Möglichkeiten an, das Verhalten der dargestellten Akteure auf universale „bekannte Sätze und Lebensregeln“3 hin auszudeuten.

Das nimmt seinen Ausgangspunkt in der antiken Rhetorik, in der die Fabel eine Untergruppe der Beispiele, der paradeígmata, bildet und als Beweismittel herangezogen werden kann, um Evidenz zu erzeugen. ${ }^{4}$ Ermittelt werden Fabelsujets, indem aus den Eigenschaften der Tiere allgemeine Lehren deduziert werden. Diese Eigenschaften sind häufig naturalistisch fundiert (dass etwa der Wolf größer und stärker ist als das Lamm), doch können sie auch topische Qualitäten darstellen, wie die sprichwörtliche Schläue des Fuchses, und zum Gegenstand fortwährender symbolischer Überformung und Narrativierung werden. ${ }^{5}$

Solche vielschichtigen Sinnbildungsprozesse sind aus der flexiblen Verbindung von Fabelerzählung, situativer Rahmung und Auslegung heraus möglich. Insbesondere sind die Fabelsammlungen und Tierepen des späten 15. und 16. Jahrhunderts durch Organisations- und Erzählverfahren gekennzeichnet, die sich das breite Funktionsspektrum sprechender Tiere zunutze machen.

2 So die gegen LESSINGS Begriff der „inneren Wahrscheinlichkeit“ der Fabel von Karlheinz STIERLE vorgeschlagene Terminologie, siehe STIERLE, Karlheinz: Geschichte als Exemplum Exempel als Geschichte. Zur Pragmatik und Poetik narrativer Texte. In: Geschichte - Ereignis Erzählung. Hrsg. von Reinhart KosELLEK/Wolf-Dieter STEMPEL, München 1973 (Poetik und Hermeneutik 5), S. 347-375, hier S. 356. Vgl. GRUBMÜLLER, Klaus: Fabel, Exempel, Allegorese. Über Sinnbildungsverfahren und Verwendungszusammenhänge. In: Exempel und Exempelsammlungen. Hrsg. von Walter HAUG/Burghart WACHINGER, Tübingen 1991 (Fortuna vitrea 2), S. 58-76, hier S. 62.

3 BREITINGER, Johann Jakob: Critische Dichtkunst, zitiert nach: LESsing, Gotthold Ephraim: Von dem Gebrauche der Tiere in der Fabel. In: Fabeln / Abhandlungen über die Fabeln. Hrsg. von Heinz RöLLEKE, Stuttgart 1981 (RUB 27), S. 105-115, hier S. 105.

4 ARISTOTELES: Rhetorik. Übersetzt und hrsg. von Gernot KraPIngER, Stuttgart 2007 (RUB 18006), II.20, S. 121-124.

5 Den poetologischen Einsatz solcher Tiertopiken untersucht RIEGER, Hannah: Die Kunst der ,schönen Worte‘. Füchsische Rede- und Erzählstrategien im Reynke de Vos (1498). Diss. Kiel 2020. Vgl. auch WeitBReCht, Julia: Theriotopik: Vormoderne Mensch-Tier-Relationen und die Epistemologie der Tiererzählung. In: Poetica 50/3-4 (2020), S. 219-237. 
Hier werden populäre antike und mittelalterliche Fabeln gesammelt und zum Teil so organisiert, dass sie auch den tierlichen Akteuren selbst in den Mund gelegt werden. Mit der hier fassbaren Ordnung des Erzählens (im Sinne einer Organisation und Distribution des Erzählten) sind zugleich auch Aspekte erzählter oder vermittelter Ordnung berührt, denn innerhalb dieser Konstruktion wird der didaktische Anspruch der Tierfabel, das sprechende Tier auf bestimmte Alltagswahrheiten und Handlungsanweisungen hin auszulegen, auf spezifische Weise reflektiert. Wenn sich nämlich Tiere gegenseitig Fabeln erzählen, in denen wiederum Tiere agieren, dann gerät ihr Status in Bewegung. Es sind sprechende und zugleich auch beredte Tiere, die nicht nur als eloquente Akteure, sondern auch als didaktisch versierte Fabelerzähler auftreten, welche gleichsam auf die ihnen eigenen, topischen Eigenschaften zugreifen können. Hier werden Erzählverfahren fassbar, die auf der grundlegenden Funktion von Fabeltieren beruhen - dass sich an ihnen allgemeine menschliche Lehren verdeutlichen lassen - und die zusätzlich eine reflexive Qualität in Bezug auf diese Funktion besitzen, weil die Tiere ihren eigenen poetologischen Status artikulieren. Wenn Tiere Tierfabeln erzählen, dann sind dies keine bloßen Exempla; vielmehr sprechen sie gleichsam über sich und werden selbstreferentiell, ohne dabei ihre topischen Qualitäten zu verlieren.

Es handelt sich um ein äußerst vielseitiges, flexibles literarisches Verfahren, das in den beiden Texten, die im Folgenden diskutiert werden sollen, noch einen zusätzlichen Twist erhält. Mittels der beredten Tiere werden darin nicht nur menschliche Redepositionen artikuliert, sondern es wird zudem eine spezifische Perspektive auf den Menschen eingenommen, der die Tiere zu Ordnungsinstanzen erhebt, wenn sie das Verhalten der Menschen evaluieren und zum Gegenstand allgemeiner Lehren machen. Es handelt sich um den eingangs schon zitierten satirischen Text Flöh Hatz / Weiber Tratz Johann Fischarts sowie Georg Rollenhagens Tierkriegsepos Froschmeuseler ${ }^{6}$.

Beide Autoren machen die antiken und mittelalterlichen Traditionszusammenhänge sprechender Tiere auf sehr unterschiedliche Weise produktiv, wie im Folgenden am jeweiligen Gebrauch der Fabel von Stadtmaus und Feldmaus gezeigt werden soll. Die Vorlage dafür bildet eine Satire des Horaz: ${ }^{7}$ Zuerst lädt die Feldmaus die Stadtmaus zu sich ein und bewirtet sie mit einfachen Speisen; daraufhin erfolgt die Gegeneinladung in die Stadt, wo die Mahlzeit viel luxuriö-

6 Zitate nach der Ausgabe: Rollenhagen, Georg: Froschmeuseler. Mit den Holzschnitten der Erstausgabe. Hrsg. von Dietmar PEIL, Frankfurt a. M. 1989 (Bibliothek der frühen Neuzeit, Abt. 1, Bd. 12; Bibliothek deutscher Klassiker 48) im Folgenden unter der Sigle FM.

7 Q. Horatius Flaccus: Satiren, Briefe = Sermones, Epistulae. Übers. v. Gerd HERRMANN, hrsg. v. Gerhard Fink, Berlin 2014, II.6, 80-117; S. 122-129. 
ser ausfällt, jedoch werden die beiden vom Menschen verjagt. Dem bescheidenen Landleben wird so die Üppigkeit des Urbanen entgegengestellt; das Stadtleben erscheint aber der Feldmaus so gefahrvoll, dass sie lieber wieder aufs Land zurückkehrt.

Diese Fabel ist in allen wichtigen lateinischen und volkssprachlichen Fabelsammlungen des Mittelalters überliefert. ${ }^{8}$ In der Auslegung wird meist von Horaz der anti-urbane Diskurs übernommen, wenn die Vorzüge eines bescheidenen, aber sicheren Lebens hervorgehoben werden, wobei der Reiz des Sujets in den Gestaltungsmöglichkeiten dieses Kontrastes und seiner jeweiligen axiologischen Besetzung liegt. Auch Fischart und Rollenhagen greifen dies in ihren Tierepen auf, wobei sie die Fabel sehr unterschiedlich situativ rahmen, auserzählen und die Lizenzen des Animalischen auf je eigene Weise dafür nutzen, einen entlarvenden Blick auf die Menschen zu richten.

\section{Johann Fischarts Flöh Hatz / Weiber Tratz ( $\left.{ }^{2} 1577\right)$}

Flöh Hatz / Weiber Tratz erschien erstmals 1573 in Straßburg und wurde von Fischart von den ursprünglich 892 Versen für die zweite Auflage von 1577 um etwa das Doppelte erweitert. ${ }^{9}$ Als satirische Gerichtsrede, in der ein Floh die

8 DiCKE, Gert/GrubmÜlLER, Klaus: Die Fabeln des Mittelalters und der frühen Neuzeit. Ein Katalog der deutschen Versionen und ihrer lateinischen Entsprechungen, München 1987 (Münstersche Mittelalter-Schriften 60), Nr. 541 („Stadtmaus und Feldmaus“), S. 617-621.

9 Auf Grundlage eines Flohliedes (Alt gemein Flöhenlied. In: ScHмIтz, Rainer: Flohwalzer, Flohfallen und Flöhe im Ohr: Ein Lesebuch, Leipzig 1997, S. 18f. nach einem Flugblatt von 1530) wurde die erste Auflage wohl maßgeblich von Matthias Holzwarth verfasst. Die Vorlagen und die Bearbeitung Fischarts in der zweiten Auflage verzeichnet HAUfFEn, Adolf: Einleitung. In: Johann Fischart: Werke. Eine Auswahl. Erster Teil. Hrsg. von Adolf HAuffen, Stuttgart 1895 (Deutsche National-Literatur. Historisch-kritische Ausgabe 18, 1. Abt.), S. X-XXII. Zu den Modifikationen Fischarts siehe ScHILling, Michael: Skeptizistische Amplifikationen des Erzählens. Fischarts Antworten auf die epistemische Expansion der Frühen Neuzeit. In: Erzählen und Episteme. Hrsg. von Beate KelLnER/Jan-Dirk MÜlLER/Peter STROHSCHNEIDER, Berlin 2011 (Frühe Neuzeit 136), S. 69-89, zu Flöh Hatz S. 71-78; sowie LuKASCHEK, Kathrin: Die Ambivalenz der Ordnung in Fischarts Bearbeitung des Flöh-Hatz, Weiber-Tratz von 1577. In: Johann Fischart, genannt Mentzer. Frühneuzeitliche Autorschaft im intermedialen Kontext. Hrsg. von Tobias Bulang unter Mitarbeit v. Sophie KNAPP. Wiesbaden 2019 (Wolfenbütteler Abhandlungen zur Renaissanceforschung 37), S. 133-155. Zu Flöh Hatz im Rahmen eines Überblicks über das Gesamtwerk siehe KÜHLMANN, Wilhelm: Johann Fischart. Leben und Werk. In: Literatur im Elsaß von Fischart bis Moscherosch: Gesammelte Studien. Hrsg. von Wilhelm KüHLMAnN/Walter Ernst SCHÄFER, Tübingen 2001, S. 1-24; siehe auch SOMMERHALDER, Hugo: Johann Fischarts Werk. Eine Einführung, Berlin 1960. 
menschlichen Frauen ob ihrer Grausamkeit anklagt, steht Flöh Hatz in der Tradition ironischer Tierenkomien und -satiren humanistischer Prägung. ${ }^{10}$ Die Flohliteratur besitzt zusätzlich die Lizenz, mit den Flöhen erzählperspektivisch in die Betten und unter die Röcke zu kriechen und detailliert zu schildern, was es dort zu sehen gibt. Damit in Zusammenhang steht der in der Forschung meist hervorgehobene misogyne Gehalt dieses Textes. ${ }^{11}$ Daher ist gerade diese spezifische Perspektive auch von Interesse für die Frage nach der Funktion beredter Tiere, anthropologische Dispositionen, etwa Geschlechterordnungen, zu verdeutlichen.

Vor diesem Hintergrund soll der Umstand näher beleuchtet werden, dass der erste Teil von Flöh Hatz durch verschiedene narrative Ebenen durchformt ist, er mündet erst nach gut 2400 Versen in die eigentliche Gerichtsrede, in welcher der Floh gegenüber Jupiter das ihm und den Seinen widerfahrene Unrecht anprangert. ${ }^{12}$ Dem vorgeschaltet ist ein umfangreicher Dialog zwischen Fliege

10 Etwa das Encomium pulicis (1519) des Caelio Calcagnini und das Pulicis Encomium Physica Ratione Tractatum (Lyon 1550) des Petrus Gallisardus. In makkaronischer Tradition steht Knickknackio, Gripholdo: Floia, Cortum versicale de Flois schwartibus, illis deiriculis, quae omnes fere Minschos, Mannos, Vveibras, Iungfras, \&c. behùppere, \& spitzibus suis schnaflis steckere \& bitere solent. Floilandia (Straßburg) 1593. In: Spätmittelalter, Humanismus, Reformation. Texte und Zeugnisse. 2. Teilband: Blütezeit des Humanismus und Reformation. Hrsg. von Hedwig HEGER, München 1978, S. 491-497. Vgl. HaYn, Hugo/GotendORF, Alfred N.: Floh-Litteratur (de pulicibus) des In- und Auslandes, vom XVI. Jahrhundert bis zur Neuzeit, O. O. 1913.

11 Insbesondere hat Hans-Jürgen BACHORSKI im Blick auf die Geschlechterordnungen des 16. Jahrhunderts die Inszenierung grotesker weiblicher Körper in Flöh Hatz analysiert; BACHORSKI, Hans-Jürgen: Von Flöhen und Frauen. Zur Konstruktion einer Geschlechterdichotomie in Johann Fischarts Floeh Haz/Weiber Traz. In: Böse Frauen - Gute Frauen. Darstellungskonventionen in Texten und Bildern der Frühen Neuzeit. Hrsg. von Ulrike GAEBEL/Erika KARTSCHOKE, Trier 2001 (Literatur, Imagination, Realität 28), S. 253-272. Die Bezüge zum zeitgenössischen Ehediskurs untersucht MÜLLER, Maria E.: Schneckengeist im Venusleib. Zur Zoologie des Ehelebens bei Johann Fischart. In: Eheglück und Liebesjoch. Bilder von Liebe, Ehe und Familie in der Literatur des 15. und 16. Jahrhunderts. Hrsg. von Maria E. MülLER, Weinheim u. a. 1988 (Ergebnisse der Frauenforschung 14), S. 155-205. Bezüge zum Ehzuchtbüchlein (1578) und zum 5. Kapitel der Geschichtklitterung erarbeitet auch RuSTERHOLz, Peter: Form und Funktion des Komischen in der Tierdichtung des 16. Jahrhunderts. In: Daphnis 7 (1978), S. 129-154, hier S. 145. Dazu auch MÜLLER, Jan-Dirk: Von der Subversion frühneuzeitlicher Ehelehre. Zu Fischarts Ehzuchtbüchlein und Geschichtklitterung. In: The Graph of Sex and the German Text: Gendered Culture in Early Modern Germany 1500-1700. Hrsg. von Lynn TaTLOCK, Amsterdam, Atlanta 1994 (Chloe 19), S. 121-156, der auf Flöh Hatz nicht eingeht. Siehe auch Auguste WACKENHEIM: L'érotisme dans le ,Flöh Hatz' de Johann Fischart. In: Revue Alsacienne de Littérature 42 (1993), S. 63-72.

12 Der zweite Teil enthält die von einem sich selbst als Flöhkanzler und Obersten Flöharzt (FH, S. 85) titulierenden Sprecher vorgetragene Erwiderung der Frauen, die dabei selbst nicht zu Wort kommen. Vgl. dazu LUKASCHEK, Ambivalenz, S. 148-152 (Anm. 9). 
und Floh. Von der Fliege nach dem Grund seines Klagens und dem kaputten Bein befragt, berichtet der Floh von seinen schlechten Erfahrungen mit den Menschenfrauen:

\begin{abstract}
Das macht ain unzarts Frauenbild /
Die wol haißt ain hart rauhes Wild /

Wiwols ain linden Belz trägt an /

Tut sie kain lindes herz doch han.
\end{abstract}

(FH 123-126)

Bereits in dieser Exposition baut Fischart Konfliktlinien auf, die quer zur Differenz von Mensch und Tier verlaufen oder diese verkehren: ${ }^{13}$ Während die Tiere nicht nur eloquent, sondern auch ungeheuer verständig, mit Einsicht und Empathie füreinander, auftreten, sind die menschlichen Frauen wilde und raue Geschöpfe, die trotz ihres weichen belzes der Welt eine unbarmherzige Haltung entgegenbringen. ${ }^{14}$

Aus der Perspektive der Insekten sind die Frauen zudem mehrfach kodiert und stehen auf der Schwelle zwischen Mensch und Tier: Als köstliches Wildbret erscheinen sie ungeheuer verlockend und sind zugleich versierte Jägerinnen der Flöhe, so dass sie im Krieg der Spezies, der hier zugleich Krieg der Geschlechter ist, als Gegnerinnen, Schlachtfeld und Beute zugleich erscheinen.

Um diese Verkehrung zu forcieren, bedient sich Fischart in parodistischer Weise heldenepischer Register, ${ }^{15}$ wenn er den Floh von seinen Abenteuern berichten lässt: Nachdem er lange Zeit ein nahrhaftes Leben bei den Mägden im Hühnerstall geführt und ainen sichern gang (FH 784) gehabt habe, sei er - offenbar ein adoleszenter Heißsporn - auf einem Hund gewissermaßen auf Aventiure ausgeritten. Auf diesem Wege sei er in eine prächtige Kammer vorgedrungen, in der er eine wunderschöne, sich entkleidende Jungfrau erblickt habe, nach deren Blut er sich seither verzehrt habe.

Demgegenüber tritt die Fliege in einer hausväterlichen Position auf und hält den Floh dazu an, Lehren aus seiner Situation zu ziehen. Seine Verletzungen seien Konsequenz seines Handelns, die er aus Mutwill, fürwiz und vor allen Dingen: schleck (FH 780 f.), also Schlemmerei, begangen habe. Um das Argu-

13 Vgl. zur „spezifische[n] fiktionale[n] Disposition des tierepischen Erzählverfahrens“ WALTENBERGER, Michael/GLÜCK, Jan/LUKASCHEK, Kathrin: Einleitung. In: Satirische Kritik und politische Reflexion in der europäischen Tierepik. Hrsg. von DENS., Berlin, Boston 2016, S. 1-9, hier S. 5.

14 Dazu im Blick auf den zeitgenössischen politischen Diskurs, LuKASCHEK, Ambivalenz, S. 140 f. (Anm. 9).

15 Auf die Tradition der Epenparodie weist Fischart mit Bezug zur ps.-homerischen Batrachomyomachie im Epilog selbst hin, vgl. SchILLING, Skeptizistische Amplifikationen, S. 71 (Anm. 9), sowie LUKASCHEK, Ambivalenz, S. 138, Anm. 15 (Anm. 9). 
ment zu untermauern, erzählt die Fliege dem Floh auch Fabeln, darunter Von Stadtmaus und Feldmaus.

Neben der von Reife und Lebensweisheit getragenen Sprecherposition der Fliege findet sich eine weitere didaktisch-belehrende Instanz in der Flohwelt, es ist der Flohvater. Da ihn, so der Floh nämlich weiter, die Lust auf junges Blut nicht losgelassen habe, habe er zunächst den Rat seiner Eltern eingeholt. Sein Vater habe ihm daraufhin erzählt (dies präsentiert Fischart in einer eingeschobenen, ausführlichen Binnenrede [FH 994-1912]), er habe selbst, als er im Alter seines Sohnes gewesen sei, versucht, an das Blut einer Jungfrau zu kommen. Dafür habe er sich mit anderen streitbaren Flohsoldaten zusammengetan, seinen Schwarze[n] Reuter[n] (FH 1633).

Diese Horde sei in die Stadt eingefallen und marodierend durch die Kirche, über den Markt und zuletzt in ein Wohnhaus hinein gezogen, wo der Flohvater auch bis in die privaten Gemächer gelangt sei. Zunächst aber hätten sie die Kirche gestürmt, wo der Vater als Hauptmann einen Aussichtspunkt von der Kanzel und aus dem Kragen des Priesters heraus eingenommen habe. Von hier aus habe er einen entlarvenden Blick auf die Menschen gerichtet:

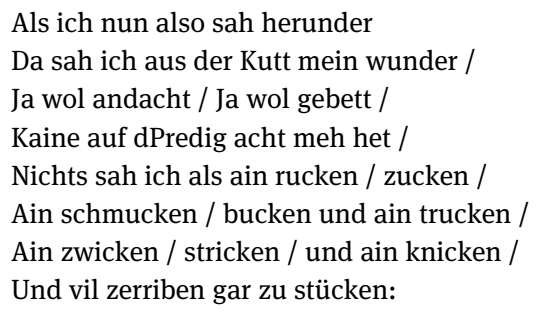

(FH 1209-1216)

Der Flohvater berichtet nun von blutigen Auseinandersetzungen, bei denen sich die Flöhe heldenmütig und tapfer gegen die Frauen zur Wehr setzen, die ihnen mit allen möglichen Listen und Gewalttätigkeiten begegnen. Dieser Krieg fordert einige Opfer, deren Nachruhm vom Flohvater besungen wird. Die FlohPerspektive erzeugt zunächst eine komische Diskrepanz von äußerst heroischem Handeln in der Selbstwahrnehmung der Flöhe und der rezipientenseitigen menschlichen Beobachtung eines Geschehens, wie es banaler nicht sein könnte: Menschen in der Kirche kratzen sich, weil die Flohstiche jucken.

Zugleich wird diese Diskrepanz von einer spezifischen Perspektivierung getragen, die aus einer subalternen Position heraus erfolgt. Die Flöhe sind nicht nur tapfer und bis zum Äußersten kampfbereit, sie kämpfen auch eine aussichtslose Schlacht gegen einen übermächtigen, überlegenen Gegner, denn es ist ein [...] gar ungleich ding / Das ain Zwerg mit aim Risen ring (FH 387 f.). Über 
den Größenunterschied zum Menschen werden die Angriffe der Flöhe zu heroischen Großtaten erklärt.

Diese animalische Perspektive birgt aber, wiederum auf der Ebene menschlicher Beobachtung, auch eine gewisse Beunruhigung: Besonderes Augenmerk verdient in diesem Kontext ein intertextueller Bezug zum Reynke de Vos, mit dem die Fliege das Kriegsgeschehen auslegt:

\begin{abstract}
Klain Leut bedörfen klaine lucken /
Gros Leut sint nicht bald zuvertrucken.

Der Raineke Fuchs kam durch ain loch

Darinn der Bruninger steckt noch:

Klain Leut bedürfen klainen Rhat /

So kommen si aus grosem schad /

Gros Leut auch grose hilf bedörfen /

Dan inn der enge sie sich schärfen /

Inn summ / das klain komt auch zustatten /

Ain klains härlin gibt auch ain schatten.
\end{abstract}

(FH 537-546)

Diese Auslegung der Fliege - dass kleine Tiere in bestimmten Situationen den größeren überlegen seien - ist, wie der schlaue Fuchs selbst, nicht ohne Tücke. Der ränkeschmiedende Reynke führt ja nichts Gutes im Schilde, wenn er den Bären Brun in einen Hinterhalt lockt. Auch dürfte Fischart und den zeitgenössischen Rezipienten bekannt gewesen sein, dass in der Erzähltradition nicht nur der Bär, sondern auch die Wölfin Hersant in einem Loch steckenbleibt - um daraufhin von Reynke hinterrücks vergewaltigt $\mathrm{zu}$ werden. ${ }^{16}$

Die geringe Größe kann also auch in aggressiver Weise gegen hochgestellte Frauen genutzt werden. Nicht nur hier erzeugt die animalische Perspektive auf menschliche Verhältnisse ein Störungsmoment oder Unbehagen, gewissermaßen ein Jucken unter der Textoberfläche, das mit den durch die

16 Darauf wird im niederdeutschen Reynke de Vos, anders als im Reinhart Fuchs, nur angespielt, wenn Ysegrym dem König die Schandtaten des Fuchses vorträgt: Dat he myn gude wyff heft gheho̊net; Reynke de Vos: Nach der Lübecker Ausgabe von 1498. Hrsg. und übertr. von Hans Joachim GeRnENTZ, Neumünster 1987, Buch II, Kap. 2, S. 18. Die Schändung der Wölfin bildet in der gesamten Erzähltradition einen der zentralen Vorwürfe gegen den Fuchs, auch wenn der Akt selbst ambivalent dargestellt und die Wölfin verschiedentlich diffamiert wird. Vgl. Hesse, Elisabeth: Der Fuchs und die Wölfin. Ein Vergleich der Hersanthandlung im Ysengrimus, im Roman de Renart und im Reinhart Fuchs. In: Schwierige Frauen - schwierige Männer in der Literatur des Mittelalters. Hrsg. von Alois Maria HAAS/Ingrid Kasten, Bern (1999), S. 111-128. 
animalische Perspektive konturierten Spannungen im sozialen Gefüge der Textwelt zu tun hat. ${ }^{17}$

Mit dieser Inszenierung des tierlichen Blicks auf die weiblichen Körper wird zum einen auf das Geschlechterverhältnis abgehoben, wenn die Flöhe - sämtlich junge, männliche und virile Tiere, gewissermaßen im besten Flohalter - weibliche Körper betrachten und $\mathrm{zu}$ erobern suchen. ${ }^{18}$ Das scheint bei Fischart zunächst kein Problem darzustellen, solange es sich um gesellschaftlich verfügbare oder nicht begehrenswerte Frauen handelt, also um Mägde und alte Frauen, die in ihrer Körperlichkeit in aggressivster Art und Weise ausgestellt werden, „monströs vergrößert und vergröbert, schmutzig und morbide, als Ansammlung von Löchern und Falten und Wölbungen, dominiert von den banalen Funktionen ihrer Körper, gesteuert allein vom mörderischen Trieb.“19

Der entlarvende Blick der Flöhe registriert jedoch zum anderen durchaus auch gesellschaftliche Differenzierungen. Als der Floh sich auf die Jagd nach der Edel Creatur (FH 2027), der begehrenswerten Jungfrau, macht, verschiebt sich die Semantik vom Kriegszug des Flohvaters zur Jagd und damit zu einem auf ein konkretes Objekt gerichteten Begehren: Die Jungfrau wird als Hase[] (FH 2018), Wildprett (FH 2025), und Luder (FH 2150) bezeichnet. Indem sich der begehrliche adoleszente Blick auf eine junge Frau in einer prächtigen Kemenate richtet, nimmt er soziale Differenzen wahr, und wenn Fischart mit voyeuristischer Lust die animalische Lizenz der Flöhe hervorhebt, Hausmauern und Kleiderschichten $\mathrm{zu}$ überwinden und somit sämtliche disziplinierenden Barrieren der menschlichen Kultur zu überschreiten, ruft er zugleich ein Drohszenario auf, in dem die schwarzen Horden die zivilisierte Sphäre überschwemmen und ins Private eindringen können.

Vor dem Hintergrund dieses Drohszenarios ist auch der abschließende Rat des Flohvaters an den Sohn zu sehen, mit dem er gewissermaßen das Epimythion zu seinem eigenen Kriegsbericht formuliert. Nach seinem Abenteuer hat der Vater den Kampf mit den Flöhhenkerin[nen] (FH 326), Flöhmörderin[nen]

17 Ohne damit Laura AUTERI darin folgen zu wollen, die Diffamierung der unteren Stände sei das zentrale Anliegen des Texts, siehe AUTERI, Laura: Tiersymbolik im deutschen Tierepos des ausgehenden 16. Jahrhunderts. In: Simpliciana 19 (1997), S. 155-165, hier S. 162.

18 „Das Frauenbild aus der Floh-Männer-Perspektive ist zutiefst ambivalent geprägt von der Spannung zwischen Libido und Sexualangst, zwischen Frauenminne und Frauenhaß. Der weiße Leib und das zarte Fleisch der Jungfrauen, die minneterminologisch gepriesen werden, erscheinen als appetitliche Leckerbissen, die zumal den erzählenden Flohhelden in einen hemmungslosen Angriffsrausch versetzen [...] Gegenüber Mägden und alten Frauen, die [...] als Objekte des Begehrens nicht in Frage kommen, bleibt nur noch die aggressive Komponente.“ MÜLLER, Schneckengeist, S. 158 (Anm. 11).

19 BACHORSKI, Von Flöhen und Frauen, S. 263 (Anm. 11). 
(FH 359) und Flöhklauberin[nen] (FH 618) für immer aufgegeben. ${ }^{20}$ Er, der angetreten war, die gesamte Damenwelt zu erobern, pocht nun auf die Einhaltung sozialer Differenz:

Du bist nicht hoher Leut genos /
Zu grosem ghört auch etwas Gros.
Pleib du bei Kundel unser Magd /
Da kanstu waiden unverjagt:

(FH 1879-1882)

An solchen Scharnierstellen, wenn das tierepische Geschehen durch unterschiedliche Instanzen kommentiert und ausgedeutet wird, nutzt Fischart nicht nur das komische Potential sprechender, sondern auch die Funktion beredter Tiere, ihr Verhalten auf „bekannte Sätze und Lebensregeln““21 hin auszudeuten.

Eine zusätzliche Verständnisebene zieht dabei die Fliege, das hausväterliche Gegenüber des Flohs, ein. Die Erzählung von den Heldentaten des Flohvaters kommentiert sie mit der Fabel von Stadtmaus und Feldmaus. Den Bezugspunkt, das Tertium, bildet hier der schleck, die Schlemmerei. Aus dem üppigen, aber gefährlichen Stadtleben zieht die Feldmaus in der Fabel den Schluss:

Mir aber will die speis nicht gfallen /
Wa schon verhonigt ist die Gallen.
Ich will liber mit sicherhait
Mein sparsamkeit und dörftigkait
Als deinen uberflus und schlecken
Mit solcher angst / sorg / flucht und schrecken.

(FH 1973-1978)

Das entspricht weitgehend dem in der antiken Vorlage vermittelten stoischen Ressentiment gegen das urbane Luxusleben. Die meisten mittelalterlichen Bearbeitungen reformulieren dies zu einem asketischen Ideal freiwilliger Armut. ${ }^{22}$

Fischart dagegen hebt, wenn er nun die erzählenden Tiere ihre Erlebnisse auslegen und so das Geschehen kommentieren lässt, die soziale Transgression

20 Während die Flöhe sprechende obszöne Eigennamen haben (vgl. BACHORSKI, Von Flöhen und Frauen, S. 264 [Anm. 11]), werden mit wenigen Ausnahmen die Frauen einzig über ihre Beziehung zu den Flöhen adressiert.

21 Vgl. Anm. 3.

22 Etwa bei Boner, Ulrich: Der Edelstein: eine mittelalterliche Fabelsammlung: zweisprachige Ausgabe. Hrsg. und übers. von Manfred Stange, Ubstadt-Weiher u. a. 2016, Nr. 15, S. 44-49 (Von einer veltmûs und einer statmûs / Von vrîer armuot), oder bei Burkard Waldis, der als Lehre formuliert: Ein armer geht on sorg da her /; Waldis, Burkard: Esopus. 400 Fabeln und Erzählungen nach der Erstausgabe von 1548. 2 Bde. Hrsg. von Ludger LIEB/Jan MoHR/Herfried VöGEL, Berlin 2011 (Frühe Neuzeit 154), Bd. 1, Nr. I,9, V. 94, S. 26. 
dessen hervor, der seinen angestammten Platz verlässt und nach Genüssen strebt, die ihm nicht zustehen. Denn nach dem Vater ermahnt nun auch die Fliege im Anschluss an die Fabel den Flohsohn: Soltst sein bei deiner Kundel pliben / Dich nicht an köstlich Leut han griben (FH $1981 \mathrm{f}$.$) .$

Die Bedeutung, die dieser Lehre beigemessen wird, zeigt sich daran, dass im zweiten Teil des Flöh Hatz durch den Flöhkanzler, also den menschlichen Sprecher der Gegenseite, ebenfalls auf die Fabel verwiesen wird. Um die Flöhe von den Maidlin (FH 3649) abzuhalten, deren Blut so süß schmecke wie Malvasir (FH 3652), wirft er ihnen soziale Anmaßung vor: Wolt wie die Feldmaus euch vermessen / Mit der Stattmaus zu nacht zuessen / (FH $3663 \mathrm{f}$.).

Fischart setzt also die Erzähl- und Auslegungsverfahren von Tierepik und Fabel gezielt ein, wenn er das Deutungspotential der bekannten Fabel auf unterschiedlichen Ebenen einlöst. Ihre Vielseitigkeit liegt in der rhetorischen Funktion begründet, ein argumentum zu unterfüttern, dessen Stoßrichtung immer kontextabhängig ist. Der Punkt, an dem Fischart ansetzt, ist eben nicht das asketische Generalverbot, sondern vielmehr die Einübung eines adäquaten und sozial verträglichen Umgangs mit männlichem Begehren. Auf der Oberfläche mag der Text sich daher spielerisch geben und sich der transgressiven Flohperspektive ungehemmt bedienen. ${ }^{23}$ Der Blick auf die jungen weiblichen Körper wird jedoch durch die Erzählsituation des Lehrdialogs als tabuisiert markiert, wenn die Tiere das Geschehen kommentierend auslegen. Die Fabel von Stadtmaus und Feldmaus verhält sich somit zu den Abenteuern des Flohvaters als Moralisatio: Sie fordert die Einhaltung derjenigen sozialen Ordnung, welche die Flöhe in ihrem triebgesteuerten Handeln permanent unterlaufen, und zeigt, dass man aus der Betrachtung derart unordentlicher Vorgänge neben voyeuristischem Vergnügen zugleich auch eine gute Lehre ziehen kann. ${ }^{24}$

23 Diese Multiperspektivität benennt auch RuSTERHOLZ, Form und Funktion, S. 144 (Anm. 11). 24 Das steht im Kontext der zeitgenössischen Satire, denn zum „Arsenal der Fischartschen Schreibtechniken“ zählt KüHLMANN auch die „mimetisch-parodistische Vorführung des Inkriminierten“, KüHLMANN, Fischart, S. 3 (Anm. 9). Auf die „Didaxe, die der Text in einem dichten Netz von Sprichwörtern, Fabeln und Exempeln ausbreitet“, verweist ScHILLING, Skeptizistische Amplifikationen, S. 76 (Anm. 9). Vgl. zu Fischarts satirischen Verfahren in der Legendenparodie auch BRockstieger, Sylvia: Literatursatire und konfessionelle Polemik. Zu Johann Fischarts Von S. Dominici und S. Francisci artlichem Leben und großen Greweln [...] (1571). In: Scientia Poetica 13 (2009), S. 21-72. 


\section{Georg Rollenhagens Froschmeuseler (1595)}

Der Froschmeuseler Georg Rollenhagens geht auf die spätantike pseudo-homerische Batrachomyomachia zurück, die vom verheerenden Krieg der Frösche mit den Mäusen handelt, doch verarbeitet Rollenhagen in drei umfangreichen Büchern zusätzlich zahlreiche antike und mittelalterliche Fabeln, Exempla und Mythen. ${ }^{25}$ Auf die Gesamtstruktur kann hier nicht eingegangen werden, der Fokus liegt im Folgenden auf dem Beginn des Ersten Buches, in dem Rollenhagen den König der Frösche, Baußback, und den Mäuseprinz Bro̊seldieb zusammentreffen lässt, die einander zunächst nicht bekämpfen, sondern lehrreiche Geschichten erzählen. ${ }^{26}$ Das übergeordnete Thema ist auch hier die Einhaltung der ständischen Ordnung, denn, so der Froschkönig Baußback:

Der ist ein weisr glůcklicher Mann /

Der sich in seim stand schicken kan.

Wer das nicht kan / der ist elend /

Vnd bleibt an Narr biß an sein end.

(FM 1439-1442)

25 Rollenhagen studierte bei Veit Oertel von Windsheim in Wittenberg, der das Werk (das der Zeit als das Homers galt) „ausdrücklich für muttersprachliche Nachdichtungen empfahl.“ KÜHLMANN, Wilhelm: Kombinatorisches Schreiben - „Intertextualität“ als Konzept frühneuzeitlicher Erfolgsautoren (Rollenhagen, Moscherosch). In: Intertextualität in der Frühen Neuzeit. Hrsg. von DEMS./Wolfgang Neuber, Frankfurt a. M. 1994 (Frühneuzeit-Studien 2), S. 111-139, hier S. 116. Aus gut 300 Hexametern macht Rollenhagen fast 20.000 Verse. Vgl. zum Verhältnis zur Vorlage LIEB, Ludger: Krieg der Sprichwörter. Zur fragwürdigen Autorität von Erfahrung und Lehre in Georg Rollenhagens Froschmeuseler. In: Tierepik und Tierallegorese. Studien zur Poetologie und historischen Anthropologie vormoderner Literatur. Hrsg. von Bernhard JAHN/ Otto NEUDECK, Frankfurt a. M. 2004 (Mikrokosmos 71), S. 251-277, hier S. 251 f. Als direkte Vorlage benennt KüHLMANN indessen das Gedicht De bello ranarum des Elisius Calentinus, das in mehreren deutschen Drucken spätestens seit 1511 vorliegt; KÜHLMANN, Kombinatorisches Schreiben, S. 135, Anm. 11 (Anm. 25). Rollenhagen hat zudem auf den Reynke in der Ausgabe mit der jüngeren protestantischen Glosse zurückgegriffen, vgl. PeIL, Dietmar: Der Einfluß des Reynke de vos auf Georg Rollenhagens Froschmeuseler. In: Reinardus 5 (1992), S. 157-169. Zu weiteren Quellen und Einflüssen siehe PEIL, Dietmar: Art. ,Rollenhagen, Georg‘. In: Enzyklopädie des Märchens. Begr. von Kurt RANKe, hrsg. von Rolf Wilhelm BREDNICH, Bd. 11, Berlin, New York 2004, Sp. 790-795, hier Sp. 792. Vgl. auch HAAS, Alois M.: Georg Rollenhagens Froschmeuseler. In: Das Tier in der Dichtung. Hrsg. von Ute SchwAB, Heidelberg 1970, S. 175-199 und S. 284-291; sowie ScHu, Sabine: Das Tierepos der Frühen Neuzeit in der Forschung seit 1980. Ein Forschungsbericht. In: Daphnis 37 (2008), S. 655-688.

26 Diese spezifische Funktionalisierung von Tieren als Fabelerzählern im Froschmeuseler untersucht KRUSE, Renke: Krieg der Spezies. Funktionalisierung von Tierdarstellungen im Froschmeuseler (1595). Diss. Kiel 2019. 
Brōseldieb hält dem entgegen: Die Meuß haben diß wol versucht (FM 1446). Die Feldmaus Warnfried sei nämlich der Stadtmaus Gutkesch auf einem Spaziergang begegnet, woraufhin sie diese zu sich nach Hause eingeladen habe. Die Fabel entspricht damit ganz dem antiken Sujet, doch wird sie nicht nur deutlich ausführlicher erzählt als die Vorlagen, sie markiert auch, indem die Akteure Namen tragen, dass diese Teil des Personals innerhalb der epischen Tierwelt sind. ${ }^{27}$

Die Einrichtung des Heims in einer Eichenwurzel ist einfach, aber behaglich: Als Gutkesch ein Stuhlpolster verlangt, gibt ihm die Dame des Hauses ein Bündel Frauenhaar, so rot wie Mohn. Der Meuse Ehrentisch (FM, Glosse zu V. 1495) besteht aus dem Schulterblatt einer Katze. Während Gutkesch sich vor all dieser rustikalen Zweckentfremdung etwas ekelt, betont der Erzähler Brôseldieb die gute Haushaltsführung mit einfachen Mitteln: Die Mahlzeit ist vom Munde abgespart, dabei gesund und nahrhaft, ${ }^{28}$ die Kinder sind sauber und wohlerzogen. Die Stadtmaus verschmäht jedoch alles, was ihr aufgetragen wird.

Demgegenüber ist der biedere Warnfried zwar skeptisch, was das Stadtleben angeht, doch beschließt er, sich die Sache erst einmal anzusehen: Was du jtzt hast hald stets fur gut / Vnd streb nach dem das besser thut (FM 1739f.). Der Übergang in die semantische Sphäre der Stadt ist nun ebenfalls, vergleichbar mit Fischart, mit einem Wechsel in eine subalterne animalische Perspektive auf die menschliche Welt verbunden. Kaum im Haus angekommen, springen die Mäuse auf die samtene Tischdecke und tun sich an den vom Hausherren übriggelassenen feinen Resten gütlich. Warnfried zeigt sich zunächst beeindruckt von diesem Englisch leben (FM 1796), doch der Schein trügt: Der Kaufmann hat $\mathrm{zu}$ viel getrunken und erwacht, weil er sich übergeben muss. Seine Frau reagiert nicht auf sein Rufen, da sie mit einem Schreiber, den sie sich ins Haus geholt hat, Hochzeit nacht (FM 1823) spielt. Es entsteht ein riesiger Tumult, Knechte und Mägde werden aufgescheucht, der Buhler entweicht durch die

27 Dieses Verfahren bezieht Peter Rusterholz auf das in französischen Poetiken der Zeit benannte Prinzip des pot-pourri, grande-salade oder fricassé. „Ein Beispiel genannter Mischpoesie“ ist für ihn die Fabel von Stadtmaus und Feldmaus, die „Fabel und Facetienliteratur“ schwankhaft vermischt; RUSTERHOLZ, Form und Funktion, S. 148 (Anm. 11). Vgl. zur politischen Funktionalisierung der Fabel im Froschmeuseler LUKASCHEK, Kathrin: Das Quaken im Schilfmeer. Ursprungsnarrativik in Rollenhagens ,Froschmeuseler‘. In: WALTENBERGER/GLÜCK/LuKASCHEK, Satirische Kritik (Anm. 13), S. 209-235.

28 Vgl. hierzu KANDLER, Johannes: „Vnser Messigkeit vns gedeyet“: Bemerkungen zum Essen und Trinken in Georg Rollenhagens Froschmeuseler. In: JAHN/NEUDECK, Tierepik und Tierallegorese (Anm. 25), S. 229-249. 
Hintertür, alles ist in Aufruhr. In der Beobachtung durch die Feldmaus erweist sich die menschliche Welt als chaotisch und sittenlos. ${ }^{29}$

Warnfried entscheidet sich nun traditionsgemäß gegen das Stadtleben, wobei seine Rückkehr in die ländliche Sphäre in auffälliger Weise erzählperspektivisch markiert wird. Auf dem Nachhauseweg trifft er am Wegesrand auf Bròseldieb, also den Erzähler:

Also kam Warnfried voller sorgen /

Wider zu Hauß am frùhen morgen.

Vnd weil ich [scil. Bro̊seldieb] da am wege saß /

Fragt ich / wie er so frühe auffwaß?

(FM 1947-1950)

Hier wird nun also die auktoriale Perspektive des Fabelerzählers in die direkte Rede Brōseldiebs überführt. Die Erzählung von Stadtmaus und Feldmaus ist nicht, wie bei Fischart, als eingeschobene raum- und zeitenthobene Fabel gestaltet, sondern als Metalepse: Brōseldieb hat die Geschichte aus erster Hand und direkt im Anschluss an Warnfrieds Erlebnisse in der Stadt erfahren. Diese Integration der Fabelhandlung ins epische Geschehen bildet ebenfalls eine Scharnierstelle, in der Handlung, Kommentar und Auslegung miteinander kurzgeschlossen werden können: „In einer tiefgestaffelten Überlagerung der Erzählebenen sind den Figuren Exkurse und moralische Exempel in den Mund gelegt, die immer wieder in Sprichworten und Sentenzen kommentiert werden.“30

Rollenhagen treibt das literarische Spiel mit den beredten Tieren noch weiter, denn auf Bròseldiebs alltägliche Frage reagiert Warnfried, anstatt direkt von seinen Abenteuern zu berichten, indem er zunächst die Fabel vom Hund beim Gastmahl erzählt. ${ }^{31}$ Wie bei Stadtmaus und Feldmaus wird auch hier die Frage verhandelt, ob es sich bei den Menschen besser oder schlechter lebe als draußen auf der Straße. Warnfried referiert also in topischer Form auf seinen kurzen, schmählichen Aufenthalt im Haus der Menschen und macht diesen zum Gegenstand allgemeiner Lebenslehre: So [scil. wie der Hund beim Gastmahl] bin auch ich zu gast gewesen / Danck Gott das ich noch bin genesen (FM 1965f.).

Auf der histoire-Ebene und gegenüber Bròseldieb bildet diese Fabel den Auftakt zu Warnfrieds Bericht. Innerhalb des discours stellt er an dieser Stelle zugleich aber einen Kommentar in Fabelform dar, der vergleichbar mit dem

29 Vgl. zur Beobachterrolle der Mäuse im Froschmeuseler KRUSE, Krieg der Spezies (Anm. 26).

30 KÜHLMANN, Kombinatorisches Schreiben, S. 119 (Anm. 25).

31 FM 1951-1964. Vgl. DiCKe/GrubMÜLLER, Fabeln des Mittelalters (Anm. 8), Nr. 298 („Hund beim Gastmahl“), S. $348 \mathrm{f}$. 
Ordnungsverfahren bei Fischart ist. Hier wird indessen nicht das Verhalten der Tiere referentialisiert, sondern im direkten Vergleich mit den Tieren das bunte Treiben der Menschen bewertet.

Die metaleptische Struktur eröffnet somit multiple Perspektiven auf die Erlebnisse Warnfrieds, aus der sich nun ganz unterschiedliche Lehren ziehen lassen. ${ }^{32}$ Für die intradiegetischen Fabelrezipienten Maus und Frosch bietet Brôseldieb eine konventionelle Auslegung an, die auf die Einhaltung der Ständeordnung abzielt. Zusätzlich aber entlarvt Warnfrieds ,Mausperspektive‘ vor allen Dingen das unmoralische Verhalten der Menschen:

O Wenn wir Meuß solten nachsagen /

Was seltzam hendel sich zutragen /

Die wir anho̊ren / vnd ansehen /

Es würd seltzam zeitung vmbgehen.

(FM 1977-1980)

Im Zentrum der Auslegung stehen damit nicht mehr Stadtmaus und Feldmaus, diese nehmen vielmehr das Manthier ${ }^{33}$, wie Rollenhagen den Menschen nennt, in den Blick und machen sich ihren eigenen Reim auf menschliche Triebhaftigkeit, Fressgier und Wollust.

\section{Ordnung des Erzählten und erzählte Ordnung: Zur Funktion ,beredter Tiere' in der Tierepik des 16. Jahrhunderts}

Flöh Hatz und Froschmeuseler, so ist deutlich geworden, stehen in einem dichten intertextuellen Geflecht aus Fabeln, Satiren und Tierepen, das hier nur angedeutet werden konnte und sich aus verschiedenen antiken, mittelalterlichen und humanistischen Traditionszusammenhängen knüpft. Topisches Tierwissen

32 RUSTERHOLz hält indessen wenig von der „allegorische[n] Überfrachtung des Geschehens durch die geschwätzig moralisierenden Mäuse und Frösche“; RusteRHOLZ, Form und Funktion, S. 149 (Anm. 11).

33 Das keins von den Manthieren wacht (FM 1748). Ursprünglich wohl von Hans Sachs „wurde die Bezeichnung Manthier offenbar eingeführt als „Fachterminus“, der erzählenden Tieren in den Mund gelegt wird.“ RuHBERG, Uwe: Das ,Manthier‘. Zur Rolle des Menschen in der Tierepik, insbesondere im Froschmeuseler Georg Rollenhagens. In: JAHN/NEUDECK, Tierepik und Tierallegorese (Anm. 25), S. 217-227, hier S. 226. 
erscheint hier gleichermaßen als ein Textwissen, als die Kenntnis von den im Tier gespeicherten Wissensbeständen und Erzählmodi.

Charakteristisch für beide Beispiele ist die Ordnung des Erzählten durch die Akkumulation und kunstvolle Verknüpfung von Fabelsujets, die sich des Potentials beredter Tiere bedienen. Fischart lässt eine Fliege die bekannte Fabel von Stadtmaus und Feldmaus erzählen, um das tierepische Geschehen zu kommentieren, während Rollenhagen seine stark erweiterte Ausgestaltung der Fabel-narratio in die tierepische Diegese integriert, um einen spezifischen animalischen - Blick in die Welt der Menschen zu werfen.

Beide Autoren machen sich das Potential beredter Tiere auf unterschiedliche Weise zunutze. Dabei wird deutlich, wie vielfältig Fabeln einsetzbar sind - nicht nur in den Möglichkeiten der Auslegung, sondern insbesondere auch in Bezug auf die Inszenierung beredter und erzählender Tiere. Diese sind zum einen in der Lage, alltagsweltliche Problemstellungen zu veranschaulichen, wie es schon in der äsopischen Fabel begegnet. Diese Funktion kann zum anderen in bestimmten Erzählsituationen ausgestellt werden, wenn die Tiere, indem sie selbst Fabeln erzählen, zugleich auch ihren poetologischen Status artikulieren und damit neue Möglichkeiten der Sinnbildung eröffnen, die man als Form der Ordnungsstiftung fassen könnte, denn so können ausgegrenzte und tabuisierte Aspekte dargestellt, benannt und auf den Menschen rückprojiziert werden.

Beide Texte nutzen dabei multiperspektivische Erzählverfahren, um aus der animalischen Distanz heraus einen schonungslosen Blick auf die Manthiere selbst zu werfen. Hierin liegt das zeitgenössische Spezifikum eines sammelnden und zugleich ordnenden Erzählens, das sich der unterschiedlichen Traditionszusammenhänge souverän $\mathrm{zu}$ bedienen weiß. Doch differieren die Beispiele darin, wie sie die Lizenzen des animalischen Blicks jeweils nutzen: Fischart verleiht den Tieren die Möglichkeit, aus ihrem eigenen Verhalten gute Lehren abzuleiten, ${ }^{34}$ während die menschlichen Frauen, grotesk vergrößert und auf ihre Körperlichkeit reduziert, bloße Projektionsfläche bleiben. Hier wird die didaktische und sozial disziplinierende Erzählsituation, also die Vermittlung und Weitergabe von väterlichen Lehren an den adoleszenten Protagonisten, betont.

Auch bei Rollenhagen sind die von den tierlichen Akteuren erzählten Fabeln als pädagogischer Kommentar zur Handlung zu verstehen, doch geht es hier insbesondere darum, aus der animalischen Perspektive einen geschärften

34 Kathrin LUKASCHEK weist (LUKASCHEK, Ambivalenz, S. 147, Anm. 55 [Anm. 9]) darauf hin, dass die Tiere aus ihren eigenen Erfahrungen nicht zu lernen vermögen, kommt aber zu dem Schluss, dass „nicht nur die Gewalt des Einzelnen wie der Masse beleuchtet wird, sondern auch die Chancen und Risiken der Listklugheit und die Notwendigkeit von Erziehung und Bildung.“ Ebd., S. 154. 
Blick auf menschliche Verhaltensweisen zu gewinnen. Für die redliche Feldmaus wird das Manthier, triebhaft, gefrässig und undiszipliniert, zum Gegenstand der Auslegung, um die seltzam zeitung (FM 1980) wenigstens aus Sicht des Tieres didaktisch fruchtbar zu machen.

Auf je unterschiedliche Weise vermessen die Texte „den Spielraum von öffentlicher und privater Klugheit auf dem Hintergrund moralischer Normenvorgaben“35 und problematisieren dabei die Reichweite, welche die so gewonnenen Lehren entwickeln können: Sie stehen beide unter einer für die Zeit charakteristischen Spannung zwischen dem Glauben an pädagogische Programme einerseits, und einer gewissen Illusionslosigkeit in Bezug auf ihren Nutzen andererseits, weil Floh, Maus und Mensch sich am Ende ja doch nicht ändern. ${ }^{36}$

Darin sind die beredten - gleichermaßen eloquenten wie didaktisch versierten - Tiere dem Menschen ähnlich und zugleich himmelweit von ihm entfernt. Aus dieser Distanz heraus können sie das Undisziplinierbare, das Verborgene und Tabuisierte ausstellen und in der intrikaten Vermittlungsform der Fabellehre gleichzeitig auch wieder didaktisieren. Für den auf diese Weise unter animalische Beobachtung gestellten Menschen [b]edeits in jedem Fall nichts guts / wan die Thir schwetzen (FH 64).

35 KÜhlmann, Kombinatorisches Schreiben, S. 119 (Anm. 25).

36 Das diskutieren Michael SchiLLING und Ludger LIEB mit unterschiedlichen Schlussfolgerungen anhand der Interdependenzen von Fabellehre und Sprichwort. Auf die „begrenzte[] Gültigkeit“ verweist SCHILLING, Skeptizistische Amplifikationen, S. 77 (Anm. 9): „Ein Sprichwort wird durch ein anderes entkräftet, die Orientierungsleistung der im Sprichwort sedimentierten Erfahrung wird zweifelhaft.“ In Bezug auf die Gesamtanlage des Froschmeuseler identifiziert Ludger LIEB dagegen eine konzeptionelle „Grundspannung“, welche aus einer „spezifischen Konstellation - höchste Dichte an geltungsgesättigter Weisheitslehre auf der einen und permanente Destruktion des Nutzens derselben Weisheitslehre auf der anderen Seite - resultiert“; LIEB, Krieg der Sprichwörter, S. 253 (Anm. 25). Vor allem durch die Aufbereitung im Register, das den Text auf unterschiedlichen Ebenen rezipierbar macht, sieht er einen grundlegenden „Glauben an die Verwertbarkeit und den positiven Nutzen all der vielen hier zusammengetragenen Puzzleteile einer humanistisch-protestantischen Bildung“ (ebd., S. 253) wirksam werden. 